\title{
Validación Preliminar del Instrumento de medición de Actitudes en Psicólogos y Estudiantes de Psicología (IAPE) ${ }^{1}$
}

\section{Preliminary Validation of the Scale of Attitudes from Psychologists and psychology students (IAPE)}

\author{
Cristóbal Guerra² \\ Luis Ulloa \\ Universidad Santo Tomás, Viña del Mar, Chile \\ Cristian Venegas \\ Universidad de Playa Ancha, Valparaíso, Chile \\ Hugo Plaza Villarroel \\ Universidad de Las Américas, Viña del Mar, Chile \\ Andrés Moltedo-Perfetti \\ Accademia dei Cognitivi della Marca, Ancona, Italia \\ (Rec: julio 2015 - Acept: octubre 2015)
}

\begin{abstract}
Resumen
La formación del psicólogo implica el abordaje tanto de competencias técnicas y específicas como el desarrollo de competencias genéricas y actitudinales. No obstante, existe escasez de instrumentos para evaluar las competencias actitudinales en población de psicólogos y estudiantes de psicología. Por esta razón el objetivo del estudio fue describir el proceso de construcción y análisis psicométrico preliminar de un instrumento de medición del perfil actitudinal en psicólogos y estudiantes de Psicología (IAPE). En las distintas fases del estudio participaron 152 estudiantes y egresados de psicología. Los participantes respondieron el IAPE y dos instrumentos para evaluar validez convergente y divergente. Los resultados indican que el instrumento final, de 17 ítems, posee una estructura unifactorial con adecuada consistencia interna. Además, los resultados dan cuenta de su validez (convergente, divergente y discriminante del instrumento). Se discute acerca de la utilidad de este instrumento en el medio nacional, a la vez que se deja claro que éste es un estudio preliminar que requiere ser replicado en el futuro.
\end{abstract}

Palabras clave: psicólogos, confiabilidad, validez, competencias genéricas.

\begin{abstract}
The process of training psychologists involves addressing both technical skills and generic or attitudinal competencies. However, there are not instruments for assessing attitudinal skills in psychologists and psychology students. Therefore, the aim of the study was to describe the process of construction and preliminary validation of an instrument of attitudinal competencies in psychologists and psychology students (IAPE). 152 students and graduates of psychology were considered in the different phases of the study. Participants answered the IAPE and another two instruments to assess convergent and divergent validity. Results showed that the final instrument consist of 17 items has one-factor structure with adequate internal consistency. Furthermore, they showed the validity (convergent, divergent and discriminant) of the instrument. Finally, it is discussed the usefulness of this instrument in the national context. At the same time it is been said that this is a preliminary study, being necessary futher researchs to conclude about IAPE validity.
\end{abstract}

Keywords: psychologists, reliability, validity, generic competencies.

\footnotetext{
1 Esta investigación forma parte del proyecto de Innovación Académica de la Universidad Santo Tomás número N000012475. Se agradece la colaboración de Andrea Fuenzalida y de Eva Dauelsberg.

2 Correspondencia a: Cristóbal Guerra Vio, Calle 1/2 Oriente 831, o cina 307, Viña del Mar, Chile. Email: cguerravio@ yahoo.es
} 


\section{Introducción}

Desde hace años se ha señalado la importancia de que las instituciones de educación superior orienten sus políticas educativas y programas académicos hacia las competencias profesionales necesarias en sus egresados (Castro, 2004). Las competencias son repertorios conductuales que integran conocimientos, rasgos de personalidad y aptitudes que influyen en el desarrollo de una tarea (Levy-Leboyer, 2003). De este modo, dentro de las competencias profesionales, se suelen señalar dos subtipos: las genéricas (asociadas a habilidades generales, útiles en cualquier profesión) y las técnicas (asociadas al conocimiento teórico y técnico específico de cada disciplina) (Beneitore, et al., 2007; Gutiérrez, 2007).

En un entorno laboral altamente competitivo, como lo es el del siglo XXI, es necesario que las universidades no sólo entreguen competencias técnicas, sino que además entreguen competencias genéricas, las cuales le permitan al futuro profesional integrarse de manera exitosa en la vida laboral (Benatuil \& Laurito, 2009). Esto es particularmente significativo para una carrera como la de Psicología, una de las que más ha crecido en Chile en los últimos años, observando un aumento de los 523 titulados/as en 1996 a 3.309 en 2012, esto es, un incremento del 633\% (Álvarez, Gómez \& Ratto, 2004; Espinoza et al., 2006; Uribe, 2004; Venegas, 2010).

Dentro del área de las competencias genéricas, resulta primordial que las distintas universidades asuman una profundización en las competencias actitudinales y valóricas durante la formación académica de pregrado, para así mantener una praxis profesional de los/as titulados/as como psicólogos/as apegada a un estricto comportamiento ético en un entorno cada vez más competitivo y desafiante. Las competencias actitudinales están referidas a elementos del "ser". Específicamente, se hayan referidas a los valores, la ética profesional, el respeto y la empatía (Aznar, Cáceres \& Hinojo, 2011).

Para la carrera de Psicología, las competencias genéricas que se aprecian como particularmente relevantes son aquellas que relaconadas con el respeto por el ser humano, así como con la ética y el compromiso con la calidad (Colegio de Psicólogos de Chile, 1999; Roe, 2003). En ese sentido, la Comisión Nacional de Acreditación de Pregrado (CNAP, 2006) señala explícitamente que las escuelas de Psicología deben incorporar en su perfil de egreso aspectos como el discernimiento ético, el respeto por la naturaleza humana y el interés por el aprendizaje, competencias que en este estudio se han denominado como "Perfil Actitudinal".
Si bien tanto desde lo teórico como desde lo institucional se observa un grado importante de consenso respecto de la relevancia de formar a los estudiantes de Psicología en el ámbito actitudinal, valórico y ético, no existen instrumentos diseñados específicamente para la evaluación de dichas variables. A nivel internacional, el único instrumento que se aproxima a lo anteriormente delineado es un cuestionario utilizado en el proyecto Tuning para medir competencias genéricas (Beneitore, et al., 2007). Sin embargo, su objetivo no consiste en la medición del perfil actitudinal de los psicólogos, sino, más bien, en evaluar las competencias genéricas en términos amplios, tal como evidenció un estudio de Orellana et al. (2007), quienes aplicaron dicho instrumento a psicólogos peruanos y concluyeron que una tarea pendiente consistía, justamente, en profundizar en las competencias genéricas en psicólogos.

A nivel nacional tampoco existen instrumentos específicos para la medición del perfil actitudinal de psicólogos o de estudiantes de psicología. El instrumento más cercano es el desarrollado recientemente por Suárez (2011), el cual evalúa ocho competencias de los psicólogos, seis de las cuales corresponden a competencias específicas y las dos restantes corresponden a competencias genéricas.

De esa manera, se considera necesaria la creación de un instrumento que sea específico para la medición del perfil actitudinal en psicólogos, puesto que podría contribuir a la mejor delimitación de las competencias genéricas de los psicólogos en formación, ayudando tanto en su formación como en la adecuación de las mallas curriculares de las universidades que dictan dicha carrera. Del mismo, modo se considera pertinente utilizar un instrumento validado en el contexto nacional, ya que es sabido que las propiedades psicométricas de los instrumentos de medida se ven afectadas por aspectos culturales (Ramírez \& Rosas, 2007).

Por esta razón, se construyó un cuestionario para evaluar el perfil actitudinal auto-percibido en estudiantes de Psicología y psicólogos. Este fue denominado IAPE. Para elaborar el instrumento se configuró un listado de aquellas actitudes que aparecían vinculadas al perfil actitudinal considerado como adecuado para un psicólogo en diversas referencias. Entre las más importantes se consideraron: el planteamiento de Roe (2003), el perfil de egreso propuesto por una escuela de Psicología chilena, las competencias de egreso sugeridas por la Comisión Nacional de Acreditación de Pregrado (2006) y las competencias genéricas propuestas en el Proyecto Tuning para América Latina y Europa (Beneitore et al., 2007; Proyecto Tuning, 2003). 
Adicionalmente, se realizó una sistematización de 69 encuestas completadas por psicólogos supervisores de prácticas profesionales finales de una escuela de psicología, donde se referían a las competencias actitudinales deseables en los estudiantes en práctica.

El resultado de la recopilación bibliográfica y de la sistematización del reporte de los supervisores de prácticas arrojó 157 competencias actitudinales. En este primer listado aparecían contempladas transcripciones literales de las actitudes referidas en las fuentes consultadas. No obstante, dado que gran parte de las 157 características apuntaban a las mismas actitudes, éstas fueron resumidas en 52 por medio del enfoque de comité (Brislin, 1980).

Las 52 competencias actitudinales fueron redactadas en formato ítem. A continuacón, estos 52 ítems iniciales fueron sometidos a validación por jueces expertos (6 psicólogos con amplia experiencia en docencia en la carrera de Psicología en distintas universidades de la V Región). Los expertos evaluaron la validez de contenido de cada reactivo. Se mantuvieron 20 de los reactivos evaluados por los 6 expertos como válidos. De esta forma se conformó la escala final (ver tabla 1).

Los 20 ítems fueron aplicados a una muestra piloto de 31 estudiantes de segundo año de Psicología. Estos estudiantes señalaron que los reactivos eran comprensibles por lo que no se hicieron nuevas modificaciones a la escala.

El objetivo de este estudio es evaluar las propiedades psicométricas del cuestionario ya referido.

\section{Método}

\section{Participantes}

En las distintas fases del estudio participaron 152 personas. 52 eran estudiantes de $1^{\circ}$ de psicología, 36 cursaban el $2^{\circ}$ año, 27 cursaban el $4^{\circ}$ año y 28 cursaban el $5^{\circ}$ año y 9 eran psicólogos ya titulados (seleccionados por los directivos de una escuela de Psicología con sede en la $\mathrm{V}$ Región por poseer un adecuado perfil actitudinal).

\section{Instrumentos}

Se utilizó una batería de tres instrumentos:

1. El Instrumento de medición de actitudes en psicólogos y estudiantes de Psicología (IAPE). Se trata de un instrumento de autoreporte de 20 reactivos.
Para responder se le pide al estudiante o psicólogo que señale en cada afirmación qué tanto lo describe como estudiante de Psicología o psicólogo. Cada ítem ofrece cuatro opciones de respuesta: (1) "no me describe nada", (2) "me describe poco", (3) "me describe moderadamente" y (4) "me describe mucho". La puntuación se obtiene de la sumatoria simple de las respuestas dadas a los ítems. A mayor puntaje obtenido por el sujeto mayor es el grado en que posee las competencias actitudinales, según su propia percepción.

2. Encuesta de competencias específicas para psicólogos (Castro, 2004). Se trata de una escala Likert de 57 ítems que miden una serie de competencias profesionales específicas de los psicólogos (p. ej., realizar un diagnóstico psicológico, realizar una intervención terapéutica o laboral). El formato de respuesta va desde 1 ("nada competente") a 5 ("muy competente"). La confiabilidad de este instrumento en el presente estudio fue adecuada (Alfa de Cronbach = 0,98).

3. Cuestionario de competencias genéricas Tuning América Latina (Beneitore et al., 2007). Se trata de un cuestionario de autorreporte que evalúa la presencia y el grado de importancia asignado a 27 competencias genéricas (p. ej., capacidad de análisis y síntesis, capacidad crítica y autocrítica, habilidad para trabajar en forma autónoma). El profesional debe responder, para cada una de las 27 afirmaciones, qué tan competente se auto percibe (desde 1 = "nada" a 4 = "mucho") y que tan importante considera dichas competencias en el ejercicio de su profesión (desde 1 = "nada" a 4 = "mucho"). La puntuación de cada sub-escala se obtiene sumando las respuestas a los 27 ítems (a mayor puntaje mayor es la percepción de competencia y de relevancia respectivamente). La confiabilidad de este cuestionario fue adecuada en este estudio tanto en la sub-escala de competencia como en la de relevancia (Alfa de Cronbach de 0,87 y 0,85 respectivamente).

\section{Procedimiento}

El proyecto fue aprobado por el comité central de una de las universidades participantes. Se solicitó consentimiento informado a los participantes. Quienes aceptaron participar lo hicieron de forma anónima.

En primer lugar, se evaluó la capacidad de discriminación de cada uno de los 20 ítems siguiendo el proceso de validación de los reactivos propuesto por Morales 
(2006). En esta fase participaron los 91 estudiantes de tercero, cuarto y quinto año. Se pidió a 30 de estos estudiantes que indicasen cómo creían que respondería un "buen psicólogo"; a otros 31 se les solicitó que respondieran como lo haría un "psicólogo regular" y a los 30 estudiantes restantes que respondieran pensando en cómo lo haría un "mal psicólogo". Se señaló que un buen psicólogo era aquel realmente comprometido con ayudar a los demás y que un mal psicólogo era aquel interesado en su propio beneficio. Se utilizó una escala para diferenciar a los buenos psicólogos (3 puntos) de los malos psicólogos (1 punto). Para evaluar si existía relación entre cada reactivo con la calidad profesional del psicólogo se realizaron análisis con $r$ de Spearman y con la prueba de Kruskal Wallis.

En segundo lugar, se realizó un análisis factorial exploratorio con el fin de determinar la estructura de la escala y se evaluó su consistencia interna mediante el cálculo del Alfa de Cronbach. Estos análisis fueron realizados con los estudiantes de primer año de psicología y con los egresados $(n=61)$.

En tercer lugar, se evaluó la validez convergente y divergente de la IAPE en la muestra de estudiantes de primer año $(n=52)$. Pare ello se evaluó la correlación $r$ de Pearson con los puntajes de instrumentos de medición de constructos teóricamente asociados (competencias genéricas) y de constructos independientes (competencias específicas).

Finalmente, se evaluó si existían diferencias entre los estudiantes de primer año $(n=52)$ y los egresados $(n=9)$. La comparación fue realizada con la prueba no paramétrica U de Mann y Whitney.

\section{Resultados}

\section{Capacidad de discriminación de los reactivos}

Como ya se explicó en la sección procedimiento, la validez fue evaluada mediante el método propuesto por Morales (2006), buscando evaluar si cada reactivo discrimina entre buenos, regulares y malos psicólogos. Como puede observarse en la tabla 1, los resultados indican que los 20 reactivos iniciales presentan validez discriminante adecuada, siendo útiles en la diferenciación de la autopercepción del perfil actitudinal de psicólogos de distinto nivel de calidad profesional. Específicamente, se observan relaciones directas y significativas entre la calidad del psicólogo y la puntuación obtenida en cada reactivo, es decir, los psicólogos descritos como de mayor calidad profesional son los que mayor puntaje obtienen en cada uno de los 20 reactivos y los descritos como de menor calidad profesional son los que menor puntaje obtienen.

Los resultados de la prueba de Kruskal Wallis (Tabla 1) confirman que existen diferencias significativas en todos los reactivos entre los psicólogos considerados como buenos, regulares y malos. En todos los casos, estos resultados indican mayores puntuaciones en los psicólogos de alta calidad profesional y menores puntuaciones en los de baja calidad profesional.

\section{Estructura factorial y consistencia interna del instrumento}

Se realizó un análisis factorial exploratorio mediante el método de ejes principales. Como se aprecia en la tabla 2 la mayoría de los reactivos carga adecuadamente en un factor único (cargas sobre 0,25 ). No obstante los ítems 3, 9 y 18 presentan cargas factoriales bajas, por lo que se decidió eliminarlos.

Para evaluar la confiabilidad del instrumento se consideraron sólo los 17 reactivos con cargas factoriales adecuadas en el factor único. Se evaluó la consistencia interna del instrumento mediante el método Alfa de Cronbach. Se eligió este procedimiento ya que requiere de una sola aplicación del instrumento y porque ha demostrado ser fidedigno cuando se trabaja con escalas de entre 3 y 20 ítems (Oviedo y Campo-Arias, 2005).

El IAPE total obtuvo un coeficiente Alfa de Cronbach de 0,76 , lo que es adecuado según el criterio de Oviedo \& Campo-Arias (2005). En la Tabla 2 se aprecia que, en general, los 17 reactivos del IAPE se encuentran relacionados con la escala total. La excepción la constituyen los ítems 8 y 14, pero la eliminación de cualquiera de ellos no aumentaría significativamente la confiabilidad de la escala. Por lo que no se sugiere la eliminación de ninguno de los 17 reactivos finales.

\section{Validez convergente y divergente de la IAPE}

Tal como sugieren Campbell y Fiske (1959), la prueba de la validez convergente del IAPE se realizó a través de la aplicación de un instrumento diseñado para medir un constructo que, teóricamente, debería estar relacionado con el perfil actitudinal de los psicólogos. Dado que las actitudes corresponden a competencias genéricas de los profesionales, se esperó obtener relaciones directas y significativas entre la puntuación del 
Tabla 1

Capacidad de discriminación de los ítems $(n=91)$

\begin{tabular}{|c|c|c|c|}
\hline & & $\begin{array}{l}\text { Relación con cali- } \\
\text { dad del psicólogo }\end{array}$ & $\begin{array}{l}\text { Diferencia entre } \\
\text { tipo de psicólogo }\end{array}$ \\
\hline 1. & Me importa comprender cómo las personas se sienten. &, $87 *$ & $67,54 * *$ \\
\hline 2. & $\begin{array}{l}\text { Creo que respetar el espacio y los tiempos del otro es fundamental, creo que } \\
\text { no corresponde invadir o presionar. }\end{array}$ & ,79* & $55,81 * *$ \\
\hline 3. & $\begin{array}{l}\text { Una de mis características como persona es que intento las cosas una y otra } \\
\text { vez hasta que consigo lo que busco. }\end{array}$ & $68^{*}$ & $43,70 * *$ \\
\hline 4. & Me siento motivado para vencer las dificultades que se me presentan. &, $81^{*}$ & $59,67 * *$ \\
\hline 5. & Me gusta innovar en mis trabajos. &, $81 *$ & $58,76 * *$ \\
\hline 6. & Cuando ocurre algo inesperado busco distintas formas para afrontarlo. &, $87 *$ & $68,37 * *$ \\
\hline 7. & $\begin{array}{l}\text { Me gusta la idea de compartir experiencias de trabajo con personas con } \\
\text { conocimientos distintos a las míos. }\end{array}$ &, $82 *$ & $61,11 * *$ \\
\hline 8. & $\begin{array}{l}\text { Me gusta ayudar a que las personas logren acuerdos cuando tienen ideas } \\
\text { distintas. }\end{array}$ &, $80^{*}$ & $57,06 * *$ \\
\hline 9. & De mis experiencias, me gusta sacar lo positivo y lo que me servirá a futuro. &, $84^{*}$ & $64,27 * *$ \\
\hline 10. & $\begin{array}{l}\text { Me informo acerca de lo que ocurre a nivel nacional e internacional en temas } \\
\text { relacionados con la Psicología. }\end{array}$ &, $80^{*}$ & $61,43 * *$ \\
\hline 11. & $\begin{array}{l}\text { Cuando trabajo con alguna persona (o cliente), me esfuerzo por comprender } \\
\text { lo que ésta necesita. }\end{array}$ &, $85^{*}$ & $65,01 * *$ \\
\hline 12. & $\begin{array}{l}\text { En mi actuar profesional, el estar frente a otro implica que en ese momento } \\
\text { él es lo más importante. }\end{array}$ &, $82 *$ & $61,03 * *$ \\
\hline 13. & $\begin{array}{l}\text { Cuando se me presentan dificultades relacionadas con mi trabajo soy capaz } \\
\text { de darme ánimo y motivarme para seguir adelante. }\end{array}$ &, $83^{*}$ & $61,32 * *$ \\
\hline 14. & Tengo claras cuáles son mis metas y sé qué hacer para lograrlas. &, $71 *$ & $45,66 * *$ \\
\hline 15. & $\begin{array}{l}\text { Cuando tengo que efectuar un trabajo o desafío importante adapto mis mé- } \\
\text { todos según sea el caso. }\end{array}$ &, $84^{*}$ & $63,64 * *$ \\
\hline 16. & $\begin{array}{l}\text { Estoy dispuesto (a) a aprender nuevas formas de desenvolverme en lo } \\
\text { profesional. }\end{array}$ &, $81 *$ & $60,96 * *$ \\
\hline 17. & $\begin{array}{l}\text { El conocer a personas que saben cosas distintas a las mías es para mí una } \\
\text { excelente oportunidad. }\end{array}$ &, $85^{*}$ & $64,43 * *$ \\
\hline 18. & Prefiero las actividades en equipo a las actividades individuales. &, $60^{*}$ & $32,44 * *$ \\
\hline 19. & Creo que cada experiencia de vida es una oportunidad para crecer. &, $83^{*}$ & $63,67 * *$ \\
\hline 20. & $\begin{array}{l}\text { Cada vez que tengo algún texto, artículo o libro de Psicología, me dan ganas } \\
\text { de leerlo e instruirme de qué se trata. }\end{array}$ &, $84 *$ & $65,46^{* *}$ \\
\hline
\end{tabular}

*r de Spearman. $p \leq 0,01 . N=91$. ** Prueba de Kruskal Wallis. $p \leq 0,01 . N$ "buenos psicólogos" = 30; $n$ "regulares psicólogos" = 31, $n$ “malos psicólogos" = 30 .

IAPE y las puntuaciones del instrumento de medición de competencias genéricas Tuning América Latina. Específicamente, se evaluó la relación entre el puntaje del IAPE, la puntuación de competencias genéricas autopercibidas $(M=80,18 ; D E=10,09)$ y la puntuación de la importancia asignada a las competencias genéricas $(M=102,13 ; D E=5,58)$ del cuestionario Tuning (Beneitore et al., 2007). Se hipotetizó que la puntuación del IAPE presentaría relaciones directas y significativas con las dos puntuaciones referidas. 
Tabla 2

Cargas factoriales y correlación ítem-Test Corregida de ítems $(n=61)$

\begin{tabular}{|c|c|c|c|c|}
\hline & Ítems escala total & $\begin{array}{l}\text { Carga } \\
\text { factorial }\end{array}$ & $\begin{array}{l}\text { Relación ítem } \\
\text { test corregida }\end{array}$ & $\begin{array}{l}\text { Alfa si elimina } \\
\text { el ítem }\end{array}$ \\
\hline 1. & Me importa comprender cómo las personas se sienten. & ,35 & ,30 & ,75 \\
\hline 2. & $\begin{array}{l}\text { Creo que respetar el espacio y los tiempos del otro es fundamental, } \\
\text { creo que no corresponde invadir o presionar. }\end{array}$ & 49 & 45 &, 74 \\
\hline 3. & $\begin{array}{l}\text { Una de mis características como persona es que intento las cosas una y } \\
\text { otra vez hasta que consigo lo que busco. }\end{array}$ &, 11 & & \\
\hline 4. & Me siento motivado por vencer las dificultades que se me presentan. &, 54 & 47 & ,73 \\
\hline 5. & Me gusta innovar en mis trabajos. & 48 & 41 &, 74 \\
\hline 6. & Cuando ocurre algo inesperado busco distintas formas para afrontarlo. &, 53 & 46 &, 74 \\
\hline 7. & $\begin{array}{l}\text { Me gusta la idea de compartir experiencias de trabajo con personas con } \\
\text { conocimientos distintos a las míos. }\end{array}$ & ,41 &, 34 &, 74 \\
\hline 8. & $\begin{array}{l}\text { Me gusta ayudar a que las personas logren acuerdos cuando tienen } \\
\text { ideas distintas. }\end{array}$ &, 27 &, 16 &, 76 \\
\hline 9. & $\begin{array}{l}\text { De mis experiencias, me gusta sacar lo positivo y lo que me servirá a } \\
\text { futuro. }\end{array}$ &, 19 & & \\
\hline 10. & $\begin{array}{l}\text { Me informo acerca de lo que ocurre a nivel nacional e internacional en } \\
\text { temas relacionados con la Psicología. }\end{array}$ & ,29 & ,26 &, 76 \\
\hline 11. & $\begin{array}{l}\text { Cuando trabajo con alguna persona (o cliente), me esfuerzo por com- } \\
\text { prender lo que ésta necesita. }\end{array}$ & ,29 &, 30 &, 75 \\
\hline 12. & $\begin{array}{l}\text { En mi actuar profesional, el estar frente a otro implica que en ese mo- } \\
\text { mento él es lo más importante. }\end{array}$ &, 36 & ,35 &, 74 \\
\hline 13. & $\begin{array}{l}\text { Cuando se me presentan dificultades relacionadas con mi trabajo soy } \\
\text { capaz de darme ánimo y motivarme para seguir adelante. }\end{array}$ &, 55 & ,46 &, 73 \\
\hline 14. & Tengo claras cuáles son mis metas y sé qué hacer para lograrlas. &, 25 &, 17 &, 76 \\
\hline 15. & $\begin{array}{l}\text { Cuando tengo que efectuar un trabajo o desafío importante, adapto mis } \\
\text { métodos según sea el caso. }\end{array}$ & 60 &, 52 &, 73 \\
\hline 16. & $\begin{array}{l}\text { Estoy dispuesto (a) a aprender nuevas formas de desenvolverme en lo } \\
\text { profesional. }\end{array}$ &, 39 &, 36 &, 75 \\
\hline 17. & $\begin{array}{l}\text { El conocer a personas que saben cosas distintas a las mías es para mí } \\
\text { una excelente oportunidad. }\end{array}$ &, 54 &, 44 &, 74 \\
\hline 18. & Prefiero las actividades en equipo a las actividades individuales. &, 19 & & \\
\hline 19. & Creo que cada experiencia de vida es una oportunidad para crecer. & 46 & 42 &, 74 \\
\hline 20. & $\begin{array}{l}\text { Cada vez que tengo algún texto, artículo o libro de Psicología, me dan } \\
\text { ganas de leerlo e instruirme de qué se trata. }\end{array}$ &, 27 &, 25 &, 75 \\
\hline
\end{tabular}

Como era de esperarse, por tratarse de constructos teóricamente convergentes, las relaciones entre la puntuación del IAPE y la puntuación de competencias genéricas fueron directas, altas y significativas (ver Tabla 3).

Por otro lado, siguiendo a Campbell y Fiske (1959), la validez divergente del IAPE fue evaluada mediante la aplicación de un instrumento destinado a medir un constructo que, teóricamente, no debiera estar relacionado con el perfil actitudinal de los psicólogos. La variable elegida para evaluar la validez divergente de la escala fue la puntuación obtenida en una encuesta de competencias específicas para psicólogos $(M=$ 128,66; $D E=55,21)$. De este modo, se hipotetizó que las puntuaciones de ambos instrumentos no estarían relacionadas pues el perfil actitudinal medido por el IAPE corresponde a competencias genéricas y no a competencias específicas. Como muestra la Tabla 3, los 
Tabla 3

Correlaciones entre la IAPE y medidas externas $(n=52)$

\begin{tabular}{cccc}
\hline $\begin{array}{c}\text { Competencias genéricas } \\
\text { autopercibidas }\end{array}$ & $\begin{array}{c}\text { Importancia } \\
\text { Competencias genéricas }\end{array}$ & $\begin{array}{c}\text { Competencia específica } \\
\text { autopercibida }\end{array}$ \\
\hline IAPE &, $56^{* *}$ &, $53^{* *}$ &, 26 \\
\hline Correlaciones $r$ de pearson. $* * p \leq 0.01$ & &
\end{tabular}

resultados apoyan esta hipótesis ya que la puntuación del IAPE no se encuentra relacionada con la puntuación de competencias específicas.

\section{Validación por grupos conocidos}

Por último, a fin de evaluar la capacidad de discriminación del IAPE, se comparó la puntuación media obtenida por estudiantes de primer año de Psicología con la obtenida por psicólogos ya titulados de la misma casa de estudios destacados por poseer un adecuado perfil actitudinal. Se hipotetizó que el instrumento sería capaz de discriminar entre ambas muestras, ya que se esperaba que los estudiantes de primer año puntuaran más bajo que los psicólogos ya titulados.

Para evaluar si existían diferencias estadísticamente significativas en los puntajes de ambas muestras, se realizaron análisis con la prueba no paramétrica $U$ de Mann y Whitney (ver Tabla 4). La comparación entre la puntuación obtenida por estudiantes de primer año y titulados indica que existen diferencias estadísticamente significativas $(\mathrm{p} \leq 0,01)$ en el IAPE $(\mathrm{U}=377,500)$. De este modo, la hipótesis de validez discriminante del IAPE recibe apoyo empírico.

\section{Tabla 4}

Media (y desviación estándar) de los estudiantes $(n=52)$ $y$ titulados $(n=9)$ en el IAPE

\begin{tabular}{ccc}
\hline & Alumnos $1^{\text {er }}$ año & Psicólogos titulados \\
\hline IAPE & $60,50(4,35)$ & $64,78(2,11)$ \\
\hline
\end{tabular}

Puntajes posibles entre 17 y 68

\section{Discusión}

En el presente artículo se describe el proceso de construcción y validación inicial de un cuestionario destinado a evaluar el perfil actitudinal en psicólogos y estudiantes de Psicología.

Después de varios análisis, los resultados dan cuenta de que la escala final está compuesta por 17 ítems (ver Tabla 5). Estos ítems presentan una estructura unifactorial y su consistencia interna es adecuada, lo que indica que sus resultados son confiables (Oviedo \& Campo-Arias, 2005).

La validez del instrumento fue evaluada mediante análisis con criterios externos. Los resultados de estos análisis avalan la validez convergente, divergente y discriminante de la escala total. Específicamente, tal y como se esperaba, la puntuación total del IAPE se asoció directa y significativamente con otras puntuaciones de competencias genéricas y no se asoció a una puntuación de competencias específicas, lo que es coherente con la teoría (Beneitore et al., 2007). Además el instrumento total discriminó adecuadamente entre estudiantes de Psicología de primer año y psicólogos ya titulados con un perfil actitudinal adecuado. Ambos resultados avalan la idea de que la puntuación total del IAPE mide de forma adecuada las actitudes de estudiantes de Psicología y psicólogos respecto de la profesión.

Pese a lo alentador de los resultados, es necesario hacer notar que el reducido tamaño de la muestra no permite su generalización. Específicamente se necesita que futuros estudios que pretendan analizar las propiedades psicométricas del IAPE lo hagan con muestras más grandes y representativas. Particularmente, es necesario señalar que el reducido tamaño de la muestra en este estudio impide el cálculo de normas de interpretación de los puntajes de la escala, por lo que este tema debiera ser abordado por futuras investigaciones.

Adicionalmente, parece pertinente considerar que la aplicación de este instrumento puede estar influida por la deseabilidad social. Esto es particularmente relevante al trabajar con estudiantes de psicología que, aun participando de forma anónima y voluntaria, pudieran asociar el acto de responder el IAPE con una instancia de evaluación y juicio de valor. Si bien esta limitación es compartida con la mayoría de las escalas actitudinales, se considera necesario relevarla a la hora de interpretar los resultados de futuras aplicaciones del IAPE. Por esta razón se sugiere que al aplicar el IAPE se intente controlar la deseabilidad social de los participantes (ya sea mediante una sensibilización previa a su aplicación, 
Tabla 5

Versión final del IAP

\begin{tabular}{|l|c|c|c|c|}
\hline $\begin{array}{l}\text { Indique que tanto cada afirmación lo (la) describe como psicólogo o es- } \\
\text { tudiante de psicología: }\end{array}$ & Nada & Poco & Moderado & Mucho \\
\hline 1. Me importa comprender cómo las personas se sienten & 1 & 2 & 3 & 4 \\
\hline $\begin{array}{l}\text { 2. Creo que respetar el espacio y los tiempos del otro es fundamental, creo } \\
\text { que no corresponde invadir o presionar }\end{array}$ & 1 & 2 & 3 & 4 \\
\hline 3. Me siento motivado por vencer las dificultades que se me presentan & 1 & 2 & 3 & 4 \\
\hline 4. Me gusta innovar en mis trabajos & 1 & 2 & 3 & 4 \\
\hline 5. Cuando ocurre algo inesperado busco distintas formas para afrontarlo. & 1 & 2 & 3 & 4 \\
\hline $\begin{array}{l}\text { 6. Me gusta la idea de compartir experiencias de trabajo con personas con } \\
\text { conocimientos distintos a las míos }\end{array}$ & 1 & 2 & 3 & 4 \\
\hline $\begin{array}{l}\text { 7. Me gusta ayudar a que las personas logren acuerdos cuando tienen } \\
\text { ideas distintas }\end{array}$ & 1 & 2 & 3 & 4 \\
\hline $\begin{array}{l}\text { 8. Me informo acerca de lo que ocurre a nivel nacional e internacional en } \\
\text { temas relacionados con la Psicología }\end{array}$ & 1 & 2 & 3 & 4 \\
\hline $\begin{array}{l}\text { 9. Cuando trabajo con alguna persona (o cliente), me esfuerzo por com- } \\
\text { prender lo que esta necesita }\end{array}$ & 1 & 2 & 3 & 4 \\
\hline $\begin{array}{l}\text { 10. En mi actuar profesional, el estar frente a otro implica que en ese } \\
\text { momento él es lo más importante. }\end{array}$ & 1 & 2 & 3 & 4 \\
\hline $\begin{array}{l}\text { 11. Cuando se me presentan dificultades relacionadas con mi trabajo soy } \\
\text { capaz de darme ánimo y motivarme para seguir adelante }\end{array}$ & 1 & 2 & 3 & 4 \\
\hline 12. Tengo claras cuáles son mis metas y sé que hacer para lograrlas & 1 & 2 & 3 & 4 \\
\hline $\begin{array}{l}\text { 13. Cuando tengo que efectuar un trabajo o desafío importante, adapto mis } \\
\text { métodos según sea el caso }\end{array}$ & 1 & 2 & 3 & 4 \\
\hline $\begin{array}{l}\text { 14. Estoy dispuesto (a) a aprender nuevas formas de desenvolverme en } \\
\text { lo profesional }\end{array}$ & 1 & 2 & 3 & 4 \\
\hline $\begin{array}{l}\text { 15. El conocer a personas que saben cosas distintas a las mías es para mí } \\
\text { una excelente oportunidad }\end{array}$ & 1 & 2 & 3 & 4 \\
\hline 16. Creo que cada experiencia de vida es una oportunidad para crecer & 1 & 2 & 3 & 4 \\
\hline $\begin{array}{l}\text { 17. Cada vez que tengo algún texto, artículo o libro de Psicología, me dan } \\
\text { ganas de leerlo e instruirme de qué se trata }\end{array}$ & 1 & 2 & 3 & 4 \\
\hline
\end{tabular}

a través la participación anónimas o en privado, o bien mediante la incorporación de algún instrumento que permita medir dicho fenómeno).

De todas formas, los resultados de este estudio preliminar permiten concluir que la versión de 17 ítems del IAPE presenta adecuadas propiedades psicométricas que avalan su uso en nuestro país.

Considerando que en Chile existe un elevado número de universidades que dictan la carrera de Psicología, el IAPE puede transformarse en una herramienta atingente, desde el ámbito investigativo y académico, para que las universidades no sólo evalúen las competencias específicas que adquieren sus profesionales a lo largo de su proceso de formación, sino que además evalúen el desarrollo de las competencias genéricas relacionadas con lo actitudinal, ético y valórico. Desde una perspectiva de responsabilidad social, se considera fundamental en el ejercicio profesional de la Psicología el desarrollo de competencias actitudinales, éticas y valóricas, pues quienes se titulan entrarán a un campo laboral en donde deberán preocuparse del bienestar de otros seres humanos. Finalmente, la formación en lo actitudinal y valórico contribuirá al ejercicio ético de la psicología y al mantenimiento de una opinión pública positiva frente a la profesión. 


\section{Referencias}

Álvarez, E., Gómez, J. \& Ratto, P. (2004). Competencias requeridas por el mercado laboral chileno y competencias actuales de estudiantes de psicología con orientación laboral- organizacional, en una universidad privada. Pharos, 11(1), 113-133. Recuperado de http://www.redalyc.org/articulo.oa?id=20811109

Aznar, I., Cáceres, M. \& Hinojo, M. (2011). La adquisición de competencias específicas en la Educación superior. Evaluando la formación del Psicopedagogo en la Universidad de Granada. Ensayos, 26, 71-93. Recuperado de https://www.revista.uclm.es/ index.php/ensayos/article/view/70

Benatuil, D. \& Laurito, J. (2009). El perfil laboral y educativo de los jóvenes psicólogos. Summa Psicológica UST, 6(2), 55-68. doi:10.18774/summa-vol6.num2-62

Beneitore, P., Esquetini, C., González, J., Marty, M., Siufi, G. \& Wagenaar, R. (2007). Reflexiones y perspectivas de la Educación Superior en América Latina. Bilbao: Publicaciones de la Universidad de Deusto.

Brislin, R. W. (1980). Translation and content analysis of oral and written material. En H. C. Triandis \& J. W. Berry (dirs.), Handbook of cross-cultural psychology (pp. 389-444). Boston: Allyn and Bacon.

Campbell, D. T. \& Fiske, D. W. (1959). Convergent and discriminant validation by the multitrait-multimethod matrix. Psychological Bulletin, 56, 81-105. Doi: 10.1037/h0046016

Castro, A. (2004). Las competencias profesionales del psicólogo y las necesidades de perfiles en los diferentes ámbitos laborales. Interdisciplinaria, 21(2), 117-152.Recuperado de http://ref.scielo. org/w6mzkf

Comisión Nacional de Acreditación de Pregrado, CNAP (2006). Criterios de evaluación de la carrera de psicología de psicología. Santiago: CNAP.

Colegio de Psicólogos de Chile (1999). Código de ética profesional. Santiago. Colegio de Psicólogos de Chile.

Espinoza, O., Fecci, E., González, L., Kluge, W., Mora, A., Ocaranza, O., Prieto, J. \& Rodríguez, E. (2006). Informe: Educación Superior en Iberoamérica el caso de Chile. Santiago: Centro Interuniversitario de Desarrollo CINDA.
Gutiérrez, J. (2007). Diseño curricular basado en competencias: manual para determinar competencias, perfiles, planes y programas de estudios. Viña del Mar: Ediciones Altazor.

Levy-Leboyer, C. (2003). Gestión de las competencias. Barcelona: Gestión 2000.

Morales, P. (2006). Medición de actitudes en psicología y educación. Madrid: Ediciones Universidad Pontificia Comillas de Madrid.

Orellana, O., García, L., Sarria, C., Morocho, J., Herrera, F., Salazar, M., Yanac, M., Rivera, J., Sotelo, L., Sotelo, N. \& Arce, F. (2007). Perfil profesional del psicólogo Sanmarquino. Revista de Investigación en Psicología, 10(2), 111-136. Recuperado de sisbib. unmsm.edu.pe/burevistas/investigacion_psicologia/V10_N2/pdf/ A08U10N2.pdf

Oviedo, H. \& Campo-Arias, A. (2005). Aproximación al uso del coeficiente alfa de Cronbach. Revista Colombiana de Psiquiatría, 34, 572-580. Recuperado de http://ref.scielo.org/yfzpws

Proyecto Tuning (2003). Listado de competencias genéricas para América latina. Recuperado de http://www.unideusto.org/tuning/ tuningal/index.php\%3Foption\%3Dcom_docman\%26task\%3Ddo $\mathrm{wn} \% 26 \mathrm{bid} \% 3 \mathrm{D} 12 \& \mathrm{rct}=\mathrm{j} \& \mathrm{frm}=1 \& \mathrm{q}=\&$ esrc $=\mathrm{s} \& \mathrm{sa}=\mathrm{U} \& \mathrm{ved}=0 \mathrm{CB}$ MQFjAAahUKEwjTq4zxs_TGAhUGWT4KHTxXC20\&usg=A FQjCNEvn5f_bjbePpmSOQpZm4isW-5vKQ

Ramírez, V. \& Rosas, R. (2007). Estandarización del WISC-III en Chile: Descripción del Test, Estructura Factorial y Consistencia Interna de las Escalas. Psykhe, 16, 91-109. Doi: 10.4067/5071822282007000100008

Roe, R. (2003). ¿Qué hace competente a un psicólogo? Papeles del psicólogo, 86, 1-12. Recuperado de www.redalyc.org/ articulo.0A? id $=77808601$

Suarez, X. (2011). Valoración de las competencias de psicología. Estudio exploratorio en muestras de estudiantes y profesionales activos. Revista de psicología de la Universidad de Chile, 20(1), 73-102. Doi: 10.5354/0719-0581.2011.13727

Uribe, D. (2004). Oferta educativa y oferta de graduados de Educación Superior. En J . Brunner \& P. Meller (Eds.). Oferta y demanda de profesionales y técnicos en Chile. (pp. 131-170). Santiago: Ril Editores.

Venegas, C. (2010). Realidad laboral de la psicología en chile y la V región: ¿Libre mercado o dignidad humana? Razón y Palabra, 72,1-56. Recuperado de www.razonypalabra.org.mx/N/N72/varia 
